\title{
Evidence for spleen dysfunction in malaria-HIV co-infection in a subset of pediatric patients
}

Regina Joice ${ }^{1}$, Charles Frantzreb ${ }^{1,2}$, Alana Pradham ${ }^{2}$, Karl B Seydel ${ }^{3,4}$, Steve Kamiza ${ }^{5}$, Dyann F Wirth ${ }^{1}$, Manoj T Duraisingh ${ }^{1}$, Malcolm E Molyneux ${ }^{5,6,7}$, Terrie E Taylor ${ }^{3,4}$, Matthias Marti ${ }^{1}$ and Danny A Milner Jr ${ }^{1,2,3}$

${ }^{1}$ Department of Immunology and Infectious Disease, The Harvard School of Public Health, Boston, MA, USA;

${ }^{2}$ Department of Pathology, The Brigham and Women's Hospital, Boston, MA, USA; ${ }^{3}$ The Blantyre Malaria Project, University of Malawi College of Medicine, Blantyre, Malawi; ${ }^{4}$ Department of Internal Medicine, College of Osteopathic Medicine, Michigan State University, East Lansing, MI, USA; ${ }^{5}$ College of Medicine, University of Malawi, Blantyre, Malawi; ${ }^{6}$ Malawi-Liverpool-Wellcome Trust Clinical Research Programme, Blantyre, Malawi and ${ }^{7}$ Liverpool School of Tropical Medicine, Liverpool, UK

The spleen has an important role in the clearance of malaria parasites, and the role of HIV co-infection on this process is yet to be described. Using a combination of histological and molecular methods, we systematically evaluated parasite load across multiple organs from HIV-positive and HIV-negative cases of an autopsy study of pediatric comatose children with malaria infection $(n=103)$ in Blantyre, Malawi. Quantification of parasite load across organs was done using histology. A subset of cases was further characterized for parasite localization and stage of development using immunohistochemistry-based labeling of parasite and host cells (5 HIV-positive, 10 HIV-negative), and quantitative RT-PCR (qRT-PCR) of asexual and sexual-specific genes (4 HIV-positive, 5 HIV-negative). The results were compared with clinical information including HIV status. The HIV-positive rate was $21 \%$ for the group studied (20 of 95 ) and HIV-positive patients had a significantly shorter duration of time between onset of illness and death, and were significantly older than HIV-negative patients. We found that spleens of HIV-positive cases had significantly higher parasite loads compared with those of HIV-negative cases in each of the three methods we used: (i) standard histology, (ii) immunohistochemistry-based labeling of Plasmodium lactate dehydrogenase (pLDH), and (iii) molecular detection of asexual parasite transcript apical membrane antigen 1 (AMA1). Immunohistochemistry-based labeling of macrophage marker CD163 in a subset of spleens revealed fewer activated macrophages containing engulfed parasites and a greater number of free unphagocytosed parasites in the HIV-positive cases. The mechanism by which HIV infection is associated with more rapid progression to severe cerebral malaria disease is possibly impairment of parasite destruction by splenic macrophages, supported by published in vitro studies showing inefficient phagocytosis of malaria parasites by HIV-infected macrophages.

Modern Pathology (2016) 29, 381-390; doi:10.1038/modpathol.2016.27; published online 26 February 2016

Cerebral malaria (CM) is caused by the parasite Plasmodium falciparum, and in children is commonly associated with seizures and coma. Autopsies of CM patients show increased brain volume and parasites sequestered within red cells in the cerebral microvasculature. ${ }^{1}$ Of patients meeting the clinical case definition of CM, a secondary classification of CM1 or CM2 can be made based on the gross and histological findings in the

Correspondence: Dr DA Milner Jr, MD, MSc, FCAP, Department of Pathology, The Brigham and Women's Hospital, 75 Francis Street, Amory 3, Boston, MA 02115, USA.

E-mail: dmilner@partners.org

Received 29 April 2015; revised 8 December 2015; accepted 21 December 2015; published online 26 February 2016 brain. ${ }^{2}$ The pathological features of CM1 are defined as having dense sequestration only, while for CM2 are defined as having in addition to cerebral sequestration, the presence of ring hemorrhages, excessive parasite pigment, and free pigment 'ghosts'. For both of these classifications, more than $20 \%$ of vessels are parasitized at autopsy and there is no other cause of death identified. One possible hypothesis is that CM1 and CM2 represent different time points of CM, ie, CM1 cases are those in which death occurs during active sequestration while in CM2 death occurs after a duration which includes schizont rupture of sufficient magnitude to produce additional histopathology. An alternative hypothesis is that the underlying pathophysiology of the two phenotypic CM states is different. In the cohort 
presented here, $\sim 60 \%$ of CM1 patients were HIV positive, suggesting a possible role of HIV on the CM1/CM2 distinction. ${ }^{3}$

The spleen is important in removing malaria parasites from the blood, and thereby controlling the infection, and yet the role of HIV co-infection in this process is yet to be described. The spleen as an organ of immune processing and filtration has the entire blood volume of a patient passed through its parenchyma numerous times per day. During a malaria infection, many early ring-stage forms of $P$. falciparum within red blood cells likewise pass through the spleen and do so unencumbered. As parasites grow and alter the surface of the host red blood cell, the spleen and its surveillance cells identify infected cells and prevent them from further circulation. The spleen is capable of retaining late-stage trophozoite stages (with dramatic surface modifications and increased host cell rigidity) as well as a fraction of earlier ring-stage parasites (with slight modifications and slightly altered deformability). ${ }^{4}$ These parasitized RBCs may be trapped and retained within the cords of the splenic red pulp, ${ }^{4,5}$ where they are then phagocytosed or pitted (removal of parasite leaving host cell intact) by resident macrophages. ${ }^{6}$ The alterations in cell surface are the basis for $P$. falciparum sequestration in the microvasculature of other tissues (brain, gut, etc), ${ }^{7,8}$ a process that enables successfully sequestered parasites to avoid destruction as they pass through the spleen.

In our cohort, patients with active malaria infections, very high parasitemia, but not severe malaria $(\mathrm{CM} 3)^{2}$ had little or no parasites present in the spleen at autopsy less than $48 \mathrm{~h}$ later. The presence of parasites in the spleen at autopsy may suggest that the malarial infection has overwhelmed the capacity of the spleen to clear parasites. The primary cell involved in this process is the macrophage, resident and circulating through the red pulp (the slow circulation of the spleen). If the ability of macrophages to phagocytose was disturbed, regardless of etiology, then we could hypothesize that the spleen's capacity would be overwhelmed and high total body parasitemia would be achieved at a rapid rate. This has in fact been observed in splenectomized individuals who have a higher parasite burden in the peripheral blood and are at higher risk of morbidity and mortality from malaria, likely due to their inability to control their parasitemia.9,10 Under this hypothesis, it would follow that some cases of CM in which the spleen's capacity is overwhelmed may be associated with a higher parasite burden in the body, including the spleen and brain, resulting in the CM1 phenotype.

As HIV prevalence is high in many malaria endemic regions, many children diagnosed with severe malaria are also HIV positive. The effect of malaria on HIV viral load is complex and variable. ${ }^{11-15}$ Some studies have shown no association with viral load and malaria status, while others demonstrated increased malarial episodes as viral load increased. More conclusive evidence exists for CD4 count, as a number of studies have demonstrated that the HIV-associated drop in CD4 count correlates with increased density of parasites, increased number of malaria complications and increased case fatality rate in patients with severe malaria. ${ }^{16-19}$ Mechanistically, HIV has been shown to impair the development of opsonizing antibodies to variant surface antigens, ${ }^{20}$ and in vitro experiments have demonstrated that HIV infection impairs phagocytosis of opsonized infected erythrocytes by macrophages. ${ }^{21}$ On the basis of these observations, we chose to test the hypothesis that among children with fatal malaria, HIV-infected patients would have a higher burden of parasites in the spleen and body overall than those without HIV. Following on our alternative hypothesis of CM1 above, we postulate that HIV could be one underlying biological condition to at least partially explain the difference between the CM categorizations.

Here, we examined a large series of autopsy cases to investigate the underlying histological differences between HIV-positive and HIV-negative individuals across CM categories. We found that HIV positivity across CM categories in this autopsy series is associated with faster progression from first symptom to death and increased asexual parasite burden across multiple organs of the body. This trend was most striking and most significant in the spleen. Further, macrophage dysfunction was apparent in the spleens of HIV-positive individuals, in particular, those with the CM1 phenotype. These findings suggest that HIV infection may be a biological contributor to the different phenotypes of CM pathology observed in our cohort, suggesting that the underlying pathobiology of the two phenotypes may be different.

\section{Materials and methods}

\section{Autopsy Series}

The autopsies analyzed in this study $(n=103)$ are part of a pediatric severe malaria and postmortem autopsy study that was performed between 1996 and 2011. ${ }^{2}$ Children meeting the clinical case definition of CM, as well as non-CM controls were admitted to the Pediatric Research Ward in the Queen Elizabeth Central Hospital in Blantyre, Malawi and enrolled in the severe malaria study upon the consent of the parent or guardian. Criteria used for diagnosis and clinical management have been previously described. $^{2}$ Autopsies were performed between 2 and $14 \mathrm{~h}$ after death. Tissue specimens were collected in $10 \%$ buffered formalin or frozen immediately to be later used for histological examination, immunohistochemistry (IHC) analysis, 
and quantitative reverse-transcriptase PCR (qRT-PCR).

The institutional review boards (IRB) at the University of Malawi College of Medicine, Michigan State University, and the Harvard School of Public Health approved all aspects of the study. Written informed consent was obtained from all parents/ guardians of the enrolled patients. IRB at the Brigham and Women's Hospital approved parts of this study including the use of discarded surgical tissue for laboratory optimization experiments.

\section{Histology}

Sections of brain, lung, heart, stomach, jejunum, ileum, colon, spleen, and skin were counted for number of parasites per 10 high powered field (hpf) as previously described. ${ }^{2,22,23}$

Sections of spleen stained with Hematoxyin and eosin were photographed at $\times 200$ with a polarizing lens. Briefly, three random $\times 200$ fields were photographed under ultra bright conditions (Light intensity $=6$; Exposure $=106 \mathrm{~ms}$ ) using a digital microscope camera (Q Color 3, Olympus America). Within ImageJ (imagej.nih.gov/ij/), each image was split into three channels and the red channel was analyzed because the pigment, under the above conditions, transmits primarily red light. An automated macro within ImageJ was written which performed the following functions for each image: (a) set a standard threshold for each image, (b) partitioned an area of the field, (c) analyzed particles for size and area, (d) repeated b and c 9 times for a total of 10 different partitions (with overlap). ${ }^{24}$ The 30 measurements (ie, 3 images $\times 10$ partitions/image) for size of particles and area occupied by particles of each case were averaged and a standard deviation was calculated. All measurements and calculations were carried out blinded to the final diagnosis.

Depigmented, Giemsa stained slides were produced as follows: unstained slides were placed in saturated alcoholic picric acid (Poly Scientific) and covered with parafilm or other airtight means for $48 \mathrm{~h}$. The slides were rinsed in $90 \%$ then $70 \%$ alcohol, washed in running tap water until the yellow color disappeared, and then stained with a Giemsa for parasites. ${ }^{25}$ Slides were then photographed without a polarizing lens at $\times 1000$ under oil immersion. Three images were taken in the red pulp of the spleen, as well as areas of red pulp adjacent to white pulp. The number of parasites present in each slide was counted using ImageJ Cellcounter. In images taken from the border of the red and white pulp, separate counts were taken for macrophages, parasites phagocytized by macrophages, and parasites outside macrophages; no macrophages were observed in the middle of the red pulp.

\section{Autopsy Tissues Used for IHC and qRT-PCR}

We performed our IHC analysis on 15 of the most recent cases for which there was a formalin-fixed paraffin-embedded (FFPE) tissue sample archived from all nine sites of interest (spleen, heart, brain, lung, liver, bone marrow, gut, kidney, and subcutaneous fat). Tissue quality assessments were previously described in a study focused on gametocyte enrichment in the same cohort. ${ }^{26}$ In that study, we started with the 13 most recent HIV-negative cases for which all 9 tissue types were available. We assessed tissue quality by performing IHC using the platelet endothelial cell adhesion molecule 1 (PECAM-1 or CD31) to label endothelial cells on three tissue types: spleen, heart, and bone marrow. For cases in which CD31 failed in at least one of the three tissues, we excluded those cases. The 10 most recently collected HIV-negative cases that met quality control criteria were used for a full organ screen using pLDH and Pfs16 and data from these are previously published. ${ }^{26}$ In the present study, we compare those published data to the five most recent HIV cases that satisfied the same criteria (all tissue types archived in FFPE, CD31 positivity in spleen, heart, and bone marrow) and collected in the same years as the previously published HIV-negative cases.

For the qRT-PCR, we analyzed RNA from a smaller subset of patients for which all tissue samples were stored in RNAlater. Since this was not done at the start of the autopsy study, only 12 total cases had this sample type available. In the previous study, ${ }^{26}$ we performed RNA quality assessments. One case was determined to have poor RNA quality as determined by lack of clear RNA bands on the Agilent Bioanalyzer. After excluding patients for which not all tissues were available or poor RNA bands, there were four HIV-positive and five HIV-negative cases.

\section{Immunohistochemistry}

FFPE tissues and control blocks were cut into 3 micron sections and mounted on slides. IHC for host markers CD163 and CD8 was performed as described previously. ${ }^{26,27}$ For parasite antigens, sections were dried overnight at $37^{\circ} \mathrm{C}$, deparaffinized in xylene, and hydrated through a series of graded alcohols, finishing in water. Antigen retrieval was performed by incubating slides at approximately $95^{\circ} \mathrm{C}$ in a steamer for $20 \mathrm{~min}$ in an EDTA solution (1 mM EDTA $+0.05 \%$ Tween at pH 8). Slides were blocked using a universal blocking buffer (Thermo Scientific) for $20 \mathrm{~min}$, followed by $10 \mathrm{~min}$ each of avidin and biotin blocking steps to block endogenous biotin and avidin, respectively. Parasite labeling was done as performed previously. ${ }^{26,28}$ Briefly, antibody incubation was then performed for $1 \mathrm{~h}$ with a mouse monoclonal antibody against Plasmodium lactate dehydrogenase (pLDH; Dr Michael Makler, FlowInc, Portland, OR, USA), diluted 1:750 in blocking buffer 
or with a mouse polyclonal anti-Pfs16 antibody (Dr. Kim Williamson, Loyola University, Chicago, IL), diluted 1:5000. Secondary anti-mouse biotinylated antibodies were used, followed by streptavidin conjugated to alkaline phosphotase (AP). Wash steps, in between each step thereafter, were performed with TBS+5\% Tween for $3 \times 5 \mathrm{~min}$. For the development of signal, naphthol phosphotase-fast red chromagen reagent was applied. Slides were subsequently rinsed in water and counterstained in Mayer's hematoxylin, and mounted in aqueous mounting medium. Slides were blinded to patient ID and independently counted by two microscopists, counting parasites in 100 consecutive high power fields, starting in the upper left corner of each section. The averages of the microscopists' counts were used for the analysis.

\section{RNA Extraction, DNAse Digest, and Reverse Transcription}

Small pieces of tissue were snap frozen and stored at $-80{ }^{\circ} \mathrm{C}$ in RNAlater in eppendorf tubes until processing. Tissue samples were processed as described previously. ${ }^{26}$ Briefly, all samples were homogenized and processed in TRI Reagent BD (Molecular Research Center) as per manufacturer instructions. RNA was cleaned up using DNAse digest (Ambion) and an additional phenol-chloroform extraction for protein removal and sample concentration. First-strand cDNA synthesis was performed using the SuperScript III First Strand Synthesis kit (Invitrogen). A total of $5000 \mu \mathrm{g}$ of RNA was input into cDNA synthesis reactions, and cDNA from $167 \mu \mathrm{g}$ of RNA input went into each quantitative PCR for those tissues for which $5000 \mu \mathrm{g}$ of RNA could be isolated. For those samples for which less than $5000 \mu \mathrm{g}$ RNA was isolated, the total amount of RNA input was recorded and final calculations were adjusted to the total RNA input per reaction.

\section{Quantitative RT-PCR}

Quantitative PCR was performed on cDNA in $20 \mu \mathrm{l}$ reaction volumes in either an ABI 7300 or an Viaa7 machine (both from Applied Biosystems). Amplification was performed using iQ SYBR Green Master Mix (Bio-Rad), and primers for stage-specific and housekeeping genes, published previously at $250 \mathrm{nM}$ concentration. ${ }^{26,29}$ Reaction conditions were as follows: 1 cycle $\times 10 \mathrm{~min}$ at $95^{\circ} \mathrm{C}$ and 40 cycles $\times 30 \mathrm{~s}$ at $95^{\circ} \mathrm{C}$ and $1 \mathrm{~min}$ at $58^{\circ} \mathrm{C}$. Each cDNA sample was run in triplicate, and each plate contained a dilution series of cDNA amplicons for each gene cloned into pGEM T-Easy plasmids (Promega). The genes included established markers with peak transcription during late asexual development: apical membrane antigen 1 (AMA1) and late gametocyte development ( $\left.P f_{S} 25\right)$, along with the constitutively expressed marker ubiquitin conjugating enzyme (UCE, PF08_0085), previously described..$^{30}$ A 100-fold dilution series ranging from $5 \times 10^{1}$ to $5 \times 10^{9}$ was run on each plate and demonstrated that each primer pair was between 90 and $110 \%$ efficient and had a detection limit ranging between $10^{1}$ and $10^{2}$ copies. The total number of transcript copies per RNA input of the sample was calculated using the CT values from a standard curve of plasmids with known concentration, and known size of plasmid and insert. The transcript copy number per sample was normalized to the total RNA input per PCR. Relative expression of the stage-specific markers was calculated as a ratio of the transcript copy number of $A M A 1$ and $P f s 25$, respectively, compared with $U C E$.

\section{Statistical Analysis}

Clinical and demographic parameters were compared between HIV-positive and HIV-negative patients using an ANOVA (parametric), Fisher's exact test (proportions), or Kruskal Wallace test (non-parametric) between CM-phenotype categories. Significant results are shown with $P$-values reported. For histology, IHC, and quantitative PCR data, non-parametric tests were used to determine significant differences in parasite marker expression in HIV-positive and HIV-negative tissues. Mann-Whitney test with Bonferroni correction was used for comparisons of histology data. Mann-Whitney tests were used for comparisons of both IHC and qRT-PCR data. Significant findings are denoted in the figures, tables, and text, with $P$-values reported. Throughout the paper, $P$-values less than 0.05 were considered as significant.

\section{Results}

\section{Diverse Clinical Phenotypes Are Represented in CM Patients}

Within the group of 103 autopsy cases of CM used in this study, we captured a wide range of clinical phenotypes on these cases, including cases from across the range of $\mathrm{CM}$ categorizations. CM was represented by $13 \mathrm{CM} 1$ and $40 \mathrm{CM} 2$ patients. Other cases included those meeting the clinical case definition of CM but dying of other non-CM causes (CM3, $n=20)$ and Other $(n=22)$ patients who did not meet the clinical case definition (including parasitemic and aparasitemic patients). When stratifying the data by CM phenotype, patients with CM1 compared with patients with CM2 had a shorter median duration between illness onset and death ( $34 \mathrm{~h}$ vs $75 \mathrm{~h}, P=0.0178$, Kruskal Wallace test), and also had higher medians of parasite density, hematocrit, platelet count, and a lower median blood lactate concentration, than CM2 individuals (Supplementary Table S1). The HIV-positive rate was significantly higher for CM1 vs CM2 (57 vs 18\%, $P=0.006$, Fisher's exact test) (Supplementary Table S1). 
Table 1 Summary of clinical parameters and tissue histology parasite counts by HIV status for patients with histologically diagnosed CM

\begin{tabular}{|c|c|c|c|}
\hline & \multicolumn{3}{|c|}{ Histologically-confirmed cerebral malaria } \\
\hline & $H I V+$ & $H I V-$ & P-value \\
\hline \multicolumn{4}{|l|}{ Demographics and history } \\
\hline Age (months) & $79(37-106)$ & $26(20-43)$ & 0.0023 \\
\hline Gender ( $\%$ male) & $47 \%$ & $51 \%$ & 0.5000 \\
\hline Onset of symptoms to death & $50(29-57)$ & $78(52-106)$ & 0.0084 \\
\hline \multicolumn{4}{|l|}{ Laboratory } \\
\hline Parasitemia $\left(\mathrm{p} \times 10^{3} / \mu \mathrm{l}\right)$ & $202(29-325)$ & $74(8-424)$ & 0.6350 \\
\hline Hematocrit (\%) & $20(18-26)$ & $20(15-26)$ & 0.7006 \\
\hline Lactate $(\mathrm{mmol} / \mathrm{l})$ & $11.1(9-12.3)$ & $11.9(5.9-15.1)$ & 0.4638 \\
\hline White cell count (cells $\times 10^{3} / \mu \mathrm{l}$ ) & $13.6(11.9-14.7)$ & $11.2(8.8-18.4)$ & 0.6334 \\
\hline Platelets (cellsx $\left.10^{3} / \mu \mathrm{l}\right)$ & $77(36-144)$ & $50(26-83)$ & 0.0592 \\
\hline \multicolumn{3}{|l|}{ Tissue histology counts ${ }^{\mathrm{a}}$} & $P$-value ${ }^{\mathrm{b}}$ \\
\hline Brain (\% vessels parasitized) & $82 \%$ & $72 \%$ & 0.0907 \\
\hline Brain (pp/100 capillaries) & $371(169-473)$ & $219(92-319)$ & 0.1289 \\
\hline Lung (pp/10 hpf) & $26(14-115)$ & $30(19-75)$ & 0.7917 \\
\hline Heart (pp/10 hpf) & $85(7-128)$ & $12(5-34)$ & 0.0110 \\
\hline Stomach (pp/10 hpf) & $116(16-567)$ & $51(13-120)$ & 0.3389 \\
\hline Jejunum (pp/10 hpf) & $188(53-1983)$ & $87(31-454)$ & 0.2428 \\
\hline Ileum (pp/10 hpf) & $110(3-276)$ & $47(13-86)$ & 0.3609 \\
\hline Colon (pp/10 hpf) & 215 (51-695) & $53(14-405)$ & 0.1904 \\
\hline Spleen (pp/10 hpf) & $170(70-222)$ & $16(4-85)$ & 0.0046 \\
\hline Skin (pp/10 hpf) & $105(0-1185)$ & $94(30-144)$ & 0.9264 \\
\hline
\end{tabular}

${ }^{\mathrm{a}}$ All continuous data shown as median [interquartile range] with Mann-Whitney statistical tests. ${ }^{\mathrm{b}}$ Mann-Whitney with Bonferroni correction for multiple testing ( 11 tests). ${ }^{\mathrm{C}}$ Tissue histology counts are in pigmented (ie, late trophozoite/schizont) parasites (pp). Italic values represent the numbers that had significant $P$-values after correction.

\section{HIV Positivity Is Associated with Faster Progression from Admission to Death}

We examined 95 patients, of whom 20 were HIV+ (21\%), out of 103 autopsies (no HIV results were available for 8 patients) (Table 1). HIV-positive patients were older than HIV-negative patients (79 vs 26 median months of age, $P=0.0023$, Mann-Whitney U). Gender and other clinical measurements that relate to severe malaria such as density of blood parasitemia, hematocrit, blood lactate concentration, white blood cell count, and platelet count showed no significant differences in means between the groups. However, the mean interval between onset of symptoms and death was significantly shorter in HIV-positive patients, who died around 2 days after onset of symptoms, as compared with over 3 days for HIV-negative patients (50 vs 78 h, $P=0.0084$, Mann-Whitney U). Since this autopsy study took place between 1996 and 2010, at the time many of these patients were enrolled in the study, CD4 counts and HIV viral loads were not being routinely measured in Malawi and certainly not in acutely-ill children. However, we obtained HIV viral load measurements for a subset of the HIV-positive cases in this study. These data come from a parallel analysis focused on the brain pathology during malaria-HIV co-infection in this same autopsy cohort. ${ }^{31}$ In the Hochman et al paper, viral loads were retrospectively measured using archived plasma and clinical charts were evaluated to determine whether these individuals met the clinical case definition of
AIDS, as according to the WHO guidelines for HIV severity. Of the $20 \mathrm{HIV}$-positive patients, 14 had plasma available for this analysis, and all 14 had quantifiable viral loads by quantitative PCR. In terms of AIDS, it was determined that all individuals had stage 1/2 (mild disease) and stage 3/4 (severe disease), but none met the clinical case criteria for AIDS. Thus, differences in malaria disease severity and progression in this cohort appear largely due to HIV infection during the mild and severe stages, but before the onset of AIDS. Notably, none of the children in this study were on anti-retroviral therapy.

\section{Greater Parasite Burden in the Spleens of HIV-Positive Individuals}

To further explore the basis of this rapid progression from symptoms to death in the HIV-malaria co-infected group, we compared the parasite burden in multiple organs in the autopsy cases. There was a higher mean parasite load in multiple organs of HIV-positive cases compared with HIV-negative cases, with differences approaching statistical significance in several organs (Table 1). These differences were most dramatic in the spleen where there was a significantly higher burden of parasites, even with multiple-corrections testing (170 vs 16 parasites per 10 high powered fields, $P=0.0046$, Mann-Whitney test).

When CM-phenotype diagnoses were considered, HIV status was unevenly distributed between classes of CM. Patients with CM1 (defined as having dense 


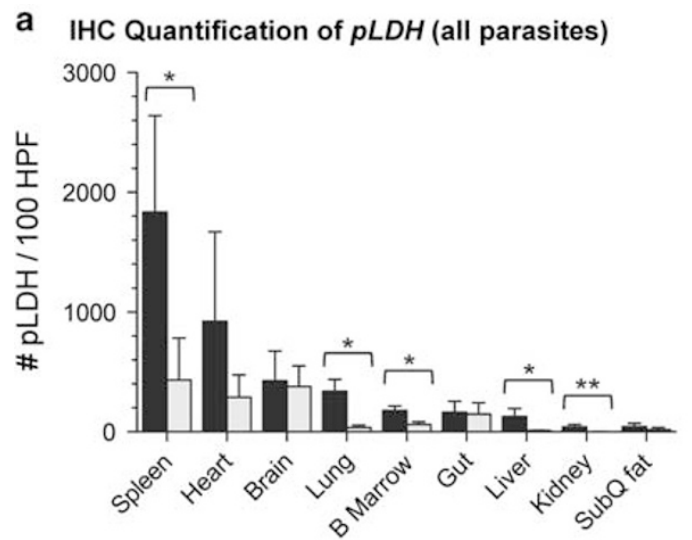

C qPCR Expn of PfAMA1 (schizonts)

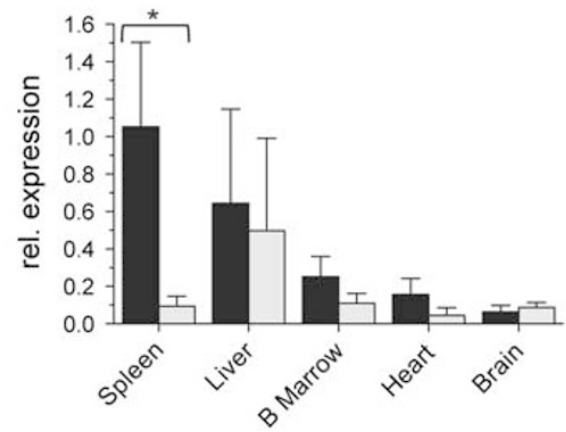

b IHC Quantification of Pfs16 (gametocytes)

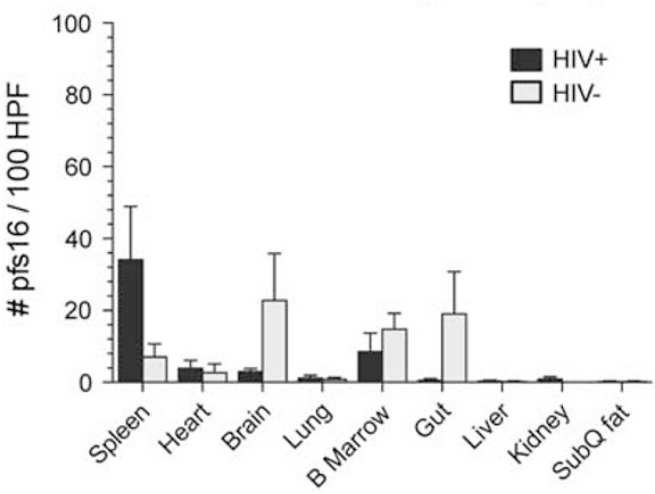

d qPCR Expn of Pfs25 (gametocytes)

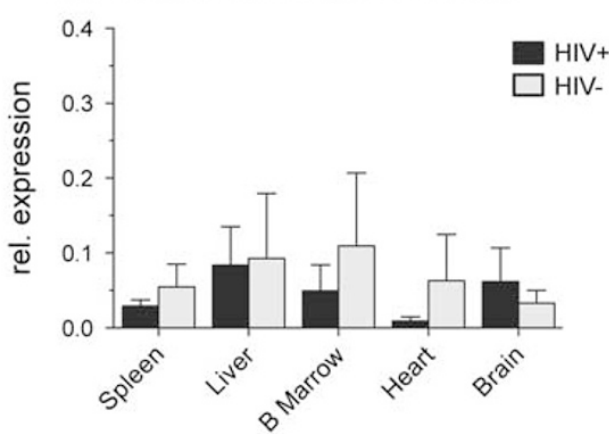

Figure 1 Increased asexual parasite burden observed across tissues in HIV+ cases. Quantities of immunohistochemistry-based (a) pLDH-labeled parasites and (b) Pfs16-labeled gametocytes quantified in $100 \mathrm{HPF}$ of tissue sections from five HIV+ (black bars) and ten HIV- (gray bars) cases reveal a significantly higher burden of parasites in the spleen, lung, bone marrow, liver and kidney tissue of HIV+ cases. The five HIV-positive cases include all nine organs. The ten HIV-negative cases include spleen, brain and bone marrow for all ten cases, and heart, lung, gut, liver, kidney and fat for six cases. Relative gene expression, as assessed by qRT-PCR of a schizont marker $(P f A M A 1, \mathbf{c})$ and a gametocyte marker $\left(P f_{s} 25, \mathbf{d}\right)$, normalized to a constitutively expressed parasite marker Ubiquitin Conjugating Enzyme (UCE) of the spleen, liver, bone marrow, heart, and brain of four HIV+ (black bars) and three HIV - cases (yellow bars) reveals a significant enrichment of $P$. falciparum asexual schizont transcripts in the spleens of HIV+ vs HIV - cases. Statistical analyses were done using Mann-Whitney test. Brackets denote comparisons in which there is a significant difference between HIV+ and HIV - marker level. Asterisks denote the $P$-value level $\left({ }^{*} P<0.05\right.$ and $\left.{ }^{*}{ }^{*} P<0.01\right)$.

sequestration without accompanying pathology) had the highest rate of HIV (57\%), with all other groups $20 \%$ or less (Supplementary Table S1). Further, as demonstrated here and in previous studies, CM1 patients had a shorter mean duration from start of illness to death, and a higher mean parasite density (Supplementary Table S1). ${ }^{3}$ In all CM categories, HIV positivity was associated with a higher mean parasite burden in the spleen, although the difference was not statistically significant in groups with few numbers. Spleen parasite burden was, however, significantly higher in CM1 HIV-positive cases as compared with CM1 HIV negatives (57 vs 24 parasites, $P=0.042$ ) (Supplementary Table S2).

Though this study does not encompass the full range of HIV disease (from mild to severe AIDS), we still did have a range of viral loads across individuals (16460-1620070 viral copies/ml with a mean of 337 391), as previously published. ${ }^{31}$ We found no significant association between HIV viral load and spleen parasitemia, though this may be due to the fact that no patients were in the most severe categories of infection (none with AIDS).

\section{Enrichment of Asexual and not Sexual Stages in the Spleens of HIV-Positive Individuals}

This enrichment of parasites in the spleen of HIV-infected patients was further confirmed by further detailed studies using stage-specific markers on a subset of these individuals for which high quality tissue and RNA was available. For a subset of cases (5 HIV+, $10 \mathrm{HIV}-$ ), we performed IHC using both the constitutive marker Plasmodium lactate dehydrogenase $(p L D H)$ and the sexual stage marker $P f s 16$ on nine organs as previously described. Tissue analyses were conducted on $10 \mathrm{HIV}$-negative cases (including spleen, brain, and bone marrow for all 10 cases, and heart, lung, gut, liver, kidney, and subcutaneous fat for 6 cases), as previously published. ${ }^{26}$ These were compared with analyses of five HIV-positive cases with all nine organs. By quantifying pLDH-labeled parasites in tissue sections from $100 \mathrm{hpf}$, we found a significantly higher parasite load in HIV patients in five of the nine organs by Mann-Whitney test (Figure 1a). The spleen was among the most significant associations, 
with spleens from HIV-positive cases having a significantly higher mean overall parasite burden than those from HIV-negative cases (1835 vs 434.2 parasites, $P=0.032$, Mann-Whitney test). The other organs with significantly higher parasite loads in HIV positives compared with HIV negatives were the lung (338.6 vs 35.5 parasites, $P=0.022$ ), bone marrow (179.1 vs 61.6 parasites, $P=0.0233$ ), liver (129 vs 10 parasites, $P=0.0135$ ), and kidney (42.23 vs 2 parasites, $P=0.0078$ ). We also quantified the number of Pfs16-labeled sexual stages (which are not typically taken up by macrophages ${ }^{32}$ ) and found that unlike pLDH, Pfs16-positive cells were not consistently higher in HIV-positive cases, and that for some tissues (brain, bone marrow, and gut), the mean number of Pfs16-positive cells was higher in HIV negative than in HIV positive. However, no statistically significant differences were found in Pfs16 levels between HIV-positive and HIV-negative subjects in any of the organs studied (Figure 1b), suggesting that asexual stages are largely responsible for the enrichment of parasites in the tissue of HIV-positive patients.

This was further confirmed by qRT-PCR from a subset of cases (4 HIV+, 5 HIV - ), comparing relative expression of asexual schizont stage marker AMA1 with that of the sexual stage marker $P f_{s} 25$ in five organs as previously described (Figure 1c and d). ${ }^{26}$ The mean expression of $\mathrm{Pfs} 25$ was lower in HIV-positive vs HIV-negative cases in the majority of tissues surveyed by qRT-PCR, though these differences were not significant, suggesting equivalent amounts of sexual stages in tissues of HIV-positive vs HIV-negative cases. On the contrary, the mean expression of AMA1 was higher in HIV-positive vs HIV-negative cases in four out of the five tissues, with the spleen of HIV-positive individuals having a significantly higher expression of the asexual schizont gene AMA1 as compared with the spleen of HIV-negative individuals (mean fold change of 1.052 vs $0.093, P=0.0357$, Mann-Whitney test). Altogether, this indicates that the observed enrichment of parasites in the organs, and specifically spleen, of HIV-positive patients is due to an increased load of the asexual stages of the parasite's life cycle.

\section{Dysfunctional Phagocytosis in the Spleens of HIV- Positive Individuals}

Depigmented Giemsa stained as well as immunohistochemically-labeled spleens showed not only higher burdens of parasites in HIV-infected patients, but also revealed distinct histological differences between the categories. The presence of 'free' parasites, ie, those not found within engulfing macrophages, was commonly observed in the spleen of HIV-positive individuals (Figure 2). Large macrophages containing parasites were more commonly observed in HIV-negative individuals (Figure 2b), while dispersed parasites outside host cells are observed in HIV-positive cases (Figure 2a) in a standard Giemsa stain. Using IHC, we observed clusters of the parasite digestion product hemozoin colocalized within CD163-labeled macrophages in HIV-negative spleens (Figure 2d). In contrast, hemozoin is very clearly localized outside host cells in HIV-positive spleens (Figure 2c). Polarizing light provided additional evidence of a pattern of scattered hemozoin representing free parasites in HIV-infected patients, while abundant pigment-aggregating macrophages were evident in HIV-negative patients (Supplementary Figure S1). Finally, immunohistochemical labeling of parasites using pLDH shows parasites localized within macrophages in HIV-negative spleens (Figure 2f), while parasites in HIV-positive spleens were observed outside macrophages (Figure 2e).

IHC labeling for CD8, a marker of macrophages as well as the littoral cell network, demonstrated that parasitized red cells in the spleen are found at the littoral cell borders. This represents the barrier back into the circulation, confirming they are in fact located in the slow circulation network of the spleen (Supplementary Figure S2). As compared with the spleens in the HIV-negative cases, the histology of the HIV-positive spleens revealed an enrichment of parasitized red cells that did not appear to be sequestered within the spleen or engulfed by macrophages.

\section{Discussion}

In this study, we set out to investigate whether HIV infection was associated with specific histological findings within CM1 and across CM categories. We found that HIV positivity, regardless of CM category, was associated with faster progression from illness onset to death, and with higher parasite burdens in the spleen as well as in other organs. This observation was recently shown by peripheral blood studies as well. ${ }^{33}$ The HIV-associated differences in parasite burden pertained specifically to the asexual fraction of the parasites; we noted no significant difference in the burden of gametocytes across these organs. We further investigated whether the increased circulating parasite density in HIV-infected subjects might be related to splenic dysfunction. Across groups, but most strikingly within the CM1 group (the group with the highest rate of HIV), we found evidence of splenic dysfunction, including enrichment of free (unphagocytosed) parasites and, conversely, a decreased numbers of parasite-containing macrophages in HIV-positive cases.

We hypothesized that when the ability of splenic macrophages to phagocytose parasitized red cells is blocked, the splenic filtration of parasitized red cells is overwhelmed, and a high total body parasitemia is achieved rapidly, akin to what is observed in splenectomized individuals. ${ }^{9,10}$ In this study we 
HIV-positive
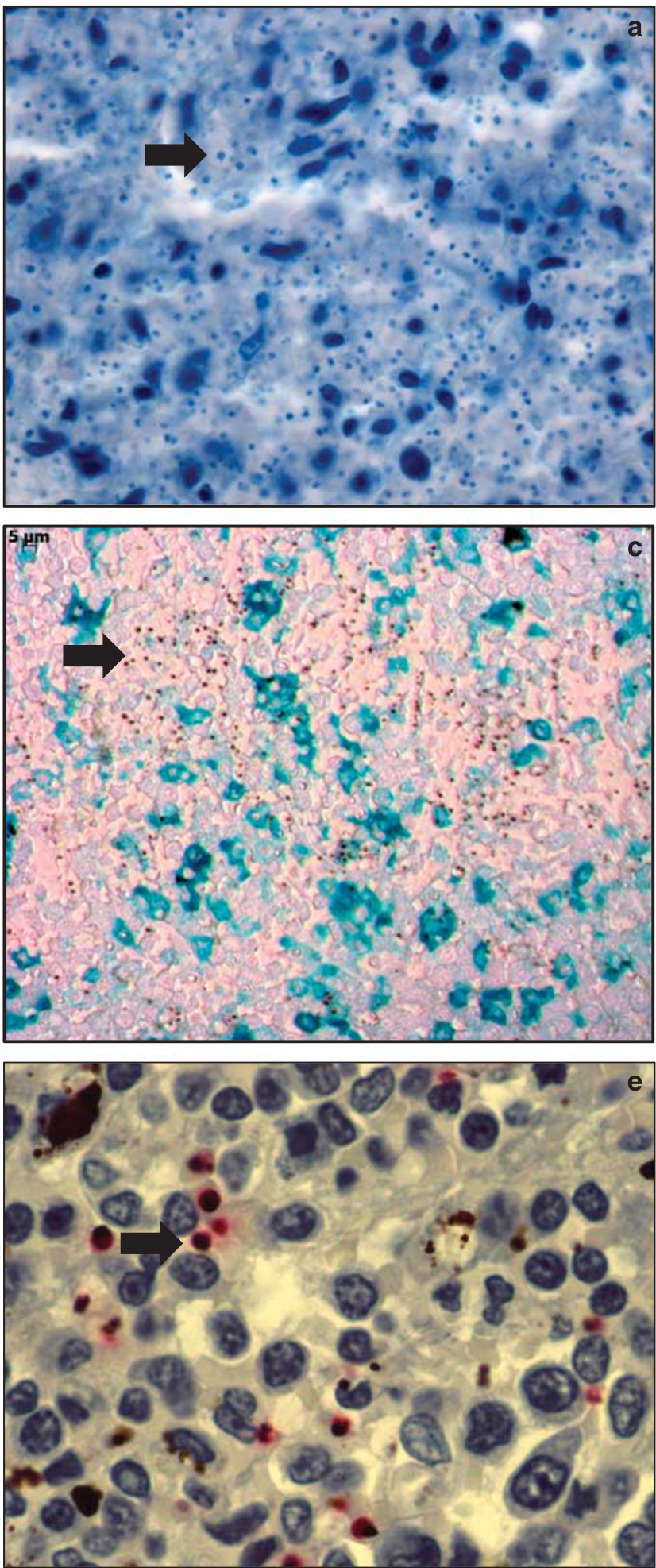

HIV-negative
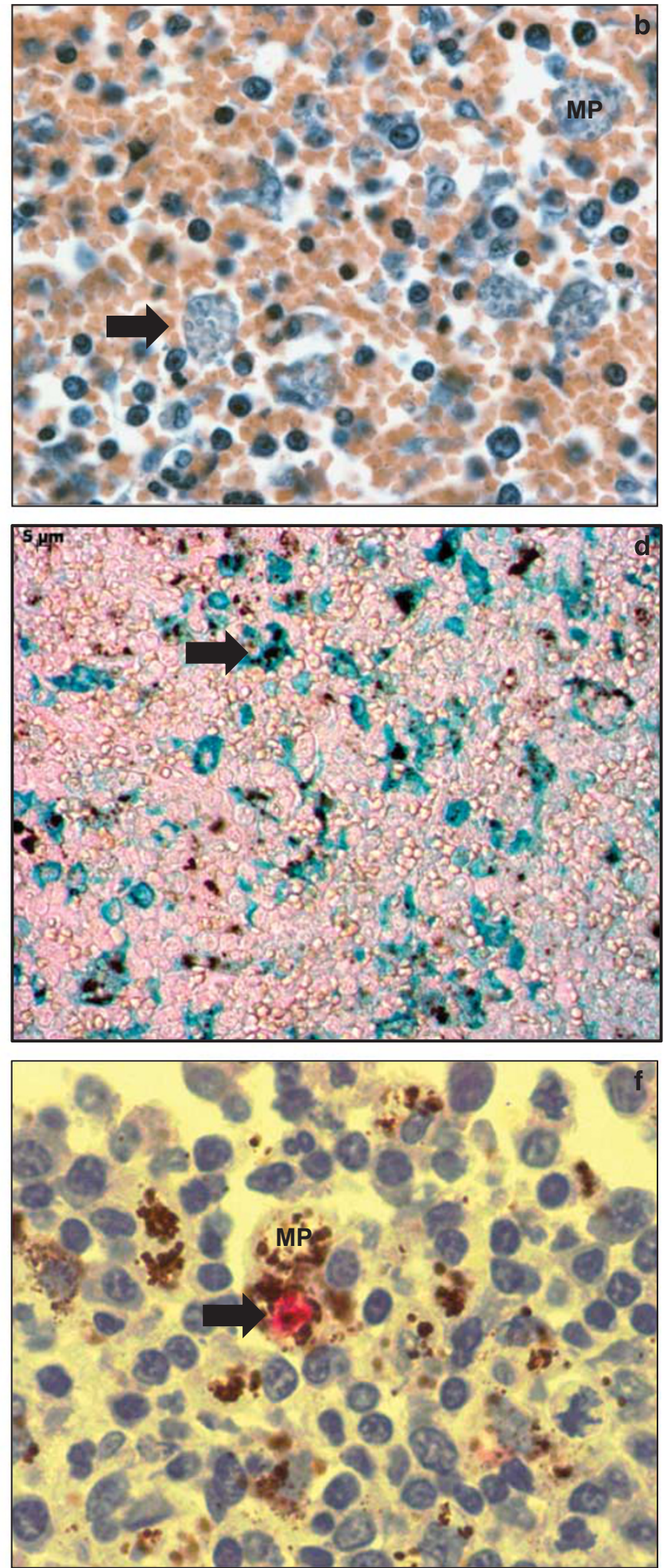

Figure 2 Histological differences in parasites in the spleen of HIV-positive vs HIV-negative patients. Image panels of histological sections from spleens of HIV-positive (a, $\mathbf{c}$ and $\mathbf{e}$ ) and HIV-negative (b, $\mathbf{d}$ and $\mathbf{f}$ ) cases. H\&E stained de-pigmented sections ( $\times 400)$ reveal a large number of free parasites, labeled in blue with hematoxylin (arrow), in the HIV+ spleen (a), compared with a large number of phagocytosed parasites (arrow) in the HIV - spleen (b) (color differences in red cells represent variability in tissue processing and staining). Immunohistochemistry with CD163 labeling (macrophages, blue) sections (shown at $\times 50$ ) also reveal free parasites (arrow) in the HIV+ spleen, as seen by small distributed hemozoin pigment (c) compared with the HIV - spleen (d) showing a large amount of clumped hemozoin phagocytosed by macrophages (shown in turquoise, arrow). Immunohistochemistry with pLDH labeling (parasites, red) and hematoxylin counterstain revealing large trophozoite-stage parasites distributed across the spleen (arrow) in HIV+ case (e), compared with a phagocytosed parasite (arrow) in a macrophage in the HIV - case (f). In panels (b) and (f) where macrophages were not labeled with a specific marker, macrophages were identified morphologically and labeled 'MP'. 
thus tested whether cases of CM in which the spleen's capacity is overwhelmed were associated with a higher parasite burden in the spleen, and body-wide.

Our findings support such a model, in that we observe what appears to be an overwhelmed spleen in CM1-HIV infection (ie, high burden of parasites in spleen and elsewhere in the body, lack of macrophages phagocytosing in spleens). In this subgroup, we observed a decrease in parasite-containing macrophages, paired with an increase in free unphagocytosed parasites in the spleens of these individuals as compared with CM1 cases who did not have HIV. Though this observation of the host-parasite histology was not found consistently with other clinical CM categories, the increased parasite load in the spleen was striking across all methodologies and range of CM-phenotype patients we examined in this study. In addition, the fact that we observed these differences to be specific to asexual parasites, which are well known to be efficiently targeted by macrophages, is also of great relevance. As sexual parasites are not efficiently taken up by macrophages, in this sense, they can be seen as a control population of malaria parasites that are presumably unaffected by macrophage-mediated immune clearance. ${ }^{26,29}$ Our data indicate that macrophage dysfunction was associated with high burdens of asexual parasites in HIV-positive patients, an observation that further supports the hypothesis that HIV is associated with macrophage dysfunction, and normal splenic immune clearance of asexual parasites. This may contribute to an accelerated progression toward severe malaria and death. We plan to do a further detailed analysis of the exact quantity, distribution, and immunophenotype of these macrophages in a future study.

From the data we present here, we conclude that HIV infection in children is associated with rapid onset of CM, and that this association may be mediated by defects in macrophage phagocytosis which diminish the spleen's ability to clear infection. As malaria endemic areas often overlap with areas in which HIV incidence is high, acceleration of CM disease by HIV is of great clinical importance. Similar mechanisms may be occurring in HIV-negative CM1 patients and warrant further study.

\section{Acknowledgments}

We would like to thank the parents and families of Malawi for their continued support of this ongoing project as well as the children of Malawi for their participation. We extend very special thanks to the indispensable team in Malawi and our US and UK collaborators that made and continues to make these autopsy tissue and clinical studies possible: George Liomba, Charles Dzamalala, Sam Wassmer, Jacqui Montgomery, Kami Kim, Sarah Hochman, Clarissa Valim, Javier Ogembo, Joyce Fingeroth,
Rob Heyderman, Dumizulu Tembo, Jimmy Vareta, Fingani Mphande, Wales Namanya, Johanness Kaliwambe, and Laston James Mbewe. This work was supported by the US National Institutes of Health (DAM: RO1 AO34969, K23 AI072033, MM: R01A1077558, TT: 5R01AI034969-14, RJ: T32 AI007535), the Harvard Initiative for Global Health, Harvard Catalyst, and by The Wellcome Trust, UK (042390/Z/94).

\section{Disclosure/conflict of interest}

The authors declare no conflict of interest.

\section{References}

1 Newton CR, Hien TT, White N. Cerebral malaria. J Neurol Neurosurg Psychiatry 2000;69:433-441.

2 Taylor TE, Fu WJ, Carr RA et al. Differentiating the pathologies of cerebral malaria by postmortem parasite counts. Nat Med 2004;10:143-145.

3 Milner D Jr, Factor R, Whitten $\mathrm{R}$ et al. Pulmonary pathology in pediatric cerebral malaria. Hum Pathol 2013;44:2719-2726.

4 Safeukui I, Correas JM, Brousse V et al. Retention of Plasmodium falciparum ring-infected erythrocytes in the slow, open microcirculation of the human spleen. Blood 2008;112:2520-2528.

5 Li J, Dao M, Lim CT, Suresh S. Spectrin-level modeling of the cytoskeleton and optical tweezers stretching of the erythrocyte. Biophys J 2005;88:3707-3719.

6 Engwerda CR, Beattie L, Amante FH. The importance of the spleen in malaria. Trends Parasitol 2005;21: 75-80.

7 MacPherson GG, Warrell MJ, White NJ et al. Human cerebral malaria. A quantitative ultrastructural analysis of parasitized erythrocyte sequestration. Am J Pathol 1985;119:385-401.

8 Seydel KB, Milner DA Jr, Kamiza SB et al. The distribution and intensity of parasite sequestration in comatose Malawian children. J Infect Dis 2006;194: 208-5.

9 Bach O, Baier M, Pullwitt et al. Falciparum malaria after splenectomy: a prospective controlled study of 33 previously splenectomized Malawian adults. Trans R Soc Trop Med Hyg 2005;99:861-867.

10 Buffet PA, Safeukui I, Deplaine G et al. The pathogenesis of Plasmodium falciparum malaria in humans: insights from splenic physiology. Blood 2011;117: 381-392.

11 Subramaniam K, Plank RM, Lin N et al. Plasmodium falciparum infection does not affect human immunodeficiency virus viral load in coinfected Rwandan adults. Open Forum Infect Dis 2014;1: ofu066.

12 Kublin JG, Patnaik P, Jere CS et al. Effect of Plasmodium falciparum malaria on concentration of HIV-1-RNA in the blood of adults in rural Malawi: a prospective cohort study. Lancet 2005;365:233-240.

13 Hoffman IF, Jere CS, Taylor TE et al. The effect of Plasmodium falciparum malaria on HIV-1 RNA blood plasma concentration. AIDS 1999;13:487-494. 
14 French N, Nakiyingi J, Lugada E et al. Increasing rates of malarial fever with deteriorating immune status in HIV-1-infected Ugandan adults. AIDS 2001;15:899-906.

15 Ariyoshi K, Schim van der Loeff M, Berry $\mathrm{N}$ et al. Plasma HIV viral load in relation to season and to Plasmodium falciparum parasitaemia. AIDS 1999;13: 1145-1146.

16 Berg A, Patel S, Aukrust $\mathrm{P}$ et al. Increased severity and mortality in adults co-infected with malaria and HIV in Maputo, Mozambique: a prospective cross-sectional study. PLoS ONE 2014;9:e88257.

17 Flateau C, Le Loup G, Pialoux G. Consequences of HIV infection on malaria and therapeutic implications: a systematic review. Lancet Infect Dis 2011;11: 541-556.

18 Hendriksen IC, Ferro J, Montoya P et al. Diagnosis, clinical presentation, and in-hospital mortality of severe malaria in HIV-coinfected children and adults in Mozambique. Clin Infect Dis 2012;55:1144-1153.

19 Sanyaolu AO, Fagbenro-Beyioku AF, Oyibo WA et al. Malaria and HIV co-infection and their effect on haemoglobin levels from three health-care institutions in Lagos, southwest Nigeria. Afr Health Sci 2013;13: 295-300.

20 Keen J, Serghides L, Ayi K et al. HIV impairs opsonic phagocytic clearance of pregnancy-associated malaria parasites. PLoS Med 2007;4:e181.

21 Ludlow LE, Zhou J, Tippett E et al. HIV-1 inhibits phagocytosis and inflammatory cytokine responses of human monocyte-derived macrophages to P. falciparum infected erythrocytes. PLoS ONE 2012;7:e32102.

22 Milner DA, Valim C, Carr RA et al. A histological method for quantifying Plasmodium falciparum in the brain in fatal paediatric cerebral malaria. Malar J 2013;12:191.

23 Milner DA Jr, Lee JJ, Frantzreb C et al. Quantitative assessment of multiorgan tissue sequestration in fatal pediatric cerebral malaria. J Infect Dis 2015;212: 1317-1321.
24 Whitten R, Milner DA Jr, Yeh MM et al. Liver pathology in Malawian children with fatal encephalopathy. Hum Pathol 2011;42:1230-1239.

25 Bancroft JD GM. Theory and Practice of Histological Techniques. 5th edn, Churchill Livingston: London, UK, 2002.

26 Joice R, Nilsson SK, Montgomery J et al. Plasmodium falciparum transmission stages accumulate in the human bone marrow. Sci Transl Med 2014;6:244re5.

27 Ogembo JG, Milner DA Jr, Mansfield KG et al. SIRPalpha/CD172a and FHOD1 are unique markers of littoral cells, a recently evolved major cell population of red pulp of human spleen. J Immunol 2012;188: 4496-4505.

28 Genrich GL, Guarner J, Paddock CD et al. Fatal malaria infection in travelers: novel immunohistochemical assays for the detection of Plasmodium falciparum in tissues and implications for pathogenesis. Am J Trop Med Hyg 2007;76:251-259.

29 Aguilar R, Magallon-Tejada A, Achtman AH et al. Molecular evidence for the localization of Plasmodium falciparum immature gametocytes in bone marrow. Blood 2014;123:959-966.

30 Daily JP, Scanfeld D, Pochet N et al. Distinct physiological states of Plasmodium falciparum in malariainfected patients. Nature 2007;450:1091-1095.

31 Hochman SE, Madaline TF, Wassmer SC et al. Fatal pediatric cerebral malaria is associated with intravascular monocytes and platelets that are increased with HIV coinfection. mBio 2015;6:e01390-15.

32 Healer J, Graszynski A, Riley E. Phagocytosis does not play a major role in naturally acquired transmissionblocking immunity to Plasmodium falciparum malaria. Infect Immun 1999;67:2334-2339.

33 Rattanapunya S, Kuesap J, Chaijaroenkul W et al. Prevalence of malaria and HIV coinfection and influence of HIV infection on malaria disease severity in population residing in malaria endemic area along the Thai-Myanmar border. Acta Trop 2015;145:55-60.

Supplementary Information accompanies the paper on Modern Pathology website (http://www.nature.com/ modpathol) 